\title{
Procrastination on Social Networks: Types and Triggers
}

\author{
Abdulaziz Alblwi, Angelos Stefanidis, Keith Phalp, Raian Ali \\ Bournemouth University, UK \\ \{aalblwi, astefanidis, kphalp, rali\}@ bournemouth.ac.uk
}

\begin{abstract}
Procrastination refers to a voluntary delay of a needed or committed task that might hurt productivity and wellbeing requirements such as self-acceptance, personal growth and positive relations with others. People might procrastinate due to a lack of motivation towards performing a task or a mismatch between the task and their skills. Social Networks Sites (SNS) are designed to provide users with the opportunity to socialise and feel relatedness despite being physically separated. SNSs design is typically equipped with a rich set of persuasive tools to encourage more social interaction. Such tools can entice procrastination when users have low self-regulation ability, and seek avoidance and mood modification. As a first step in designing persuasive features which would help people control their procrastination, there is a need to understand how existing SNS features persuade procrastination in the first place. In this paper, we conduct a multi-stage qualitative study to explore the occurrence of procrastination and the prominent features of SNS design that trigger and facilitate it. We use focus groups to explore SNS procrastination, and then get a more ecologically valid, detailed and in-context data via a ten days' diary study followed by clarification interviews. The findings shed light on prominent types of procrastination and features in SNS design that can persuade and increase, in certain contexts, the chance for procrastination.
\end{abstract}

\section{Keywords-Procrastination, Social Networking design}

\section{INTRODUCTION}

Procrastination refers to the voluntary engagement in alternative activities, which prevents the performance of other tasks, potentially leading to negative consequences [1]. Procrastination can also be defined as the postponement of a relevant and timely activity [2]. The delay caused by procrastination is associated with negative consequences such as low academic performance, reduced self-respect, and increased levels of frustration and anxiety, either for the person procrastinating or others connected to them [1]. Procrastination is considered one of the main sources of work-related stress [3]. A typical example of procrastination in an academic setting involves students delaying the start of their assignment work and, consequently, delivering low-quality work or late work, causing themselves avoidable stress.

Elsewhere, it has been demonstrated that procrastination is a coping strategy that helps people to release stress and refresh their mood, thereby positively improving work efficiency [4]. However, we argue that such kind of procrastination is a managed one, where people still enjoy a high degree of selfcontrol. Unmanaged procrastination refers to the inability to retain control over the postponement of other committed and needed tasks. In unmanaged procrastination, people deviate from what they initially planned and that mood refreshment turns into stress and low performance [5].

Social Networks Sites (SNS) platforms enable users to create personal profiles, communicate and get in touch with others regardless of their location, experiences or languages. Despite these benefits, some usage styles may turn to be harmful such as the obsessive and hasty usage that can be seen as a Digital Addiction [6]. Procrastination is a facet of such problematic usage style. SNSs are deliberately designed to persuade further user engagement. This is achieved by a range of persuasion and influence features, such as profiling, where people realize their ideal self-online or create another presence and another incarnation of their "real self" [7]. SNS also provide a medium for peer pressure, a powerful persuasive technique where users feel the urge to engage, not necessarily because of the gain of specific goals, but rather because of the fear of missing out or losing popularity [8]. This introduces the question of whether SNS design can be deemed responsible for persuading procrastination, and if so, in what way. It also raises the question of whether future SNS design can embed features which persuade and aid users to gain more control of their usage, hence gaining a higher degree of self-regulation.

Advances in persuasive technology and gamification can be exploited in SNS to gain more control over their procrastination. Persuasive technology is used to help change people's behaviour by influence rather than hard coercion and pressure [9]. Hence, using these techniques will be in harmony with the spirit of SNS that is based on freedom of choice and leisure. Gamification is a useful technique that can be used within and outside SNS to help users gain more control over procrastination. For example, when a user procrastinates because their job is not enjoyable or frustrating, it can be possible to gamify the job being undertaken to bring the joy that user experience on SNS into tasks environment. It can be used within SNS as well through goal settings and selfmonitoring, for example, a progress bar showing the daily limit and busy times of the day. A change in the SNS colour scheme in the social network to indicate an excess of the time and level of engagement would be another example.

In this paper, we study how procrastination occurs on SNS and the role of SNS features in facilitating and persuading it. Initially, we conduct a focus group study to explore the main themes and arguments. In the second phase, we use a diary study, based on the focus group findings, to elaborate and analyse on the set of initial findings. The use of a diary study helped us to get more ecologically valid, detailed and contextualized input from the participants. All participants in both studies self-declared to procrastinate often on SNS. The 
findings are meant to inform the literature on how SNS facilitate procrastination as a first step to designing persuasive techniques to counterbalance that side-effect. Hence, we used our findings in [10] as a baseline for devising countermeasures to combat procrastination on SNS.

\section{RESEARCH METHOD}

We followed a qualitative method to understand the nature of the role of SNS feature design in persuading users to procrastinate. The study was divided into different phases including a focus group, diary study and follow-up interviews in order to increase the coverage and the credibility of the study. The first phase focus group collected the initial findings and then 10 days were set aside to collect the diaries. Finally, follow-up interviews were used to clarify the data.

\section{A. Focus groups for an initial exploration}

In the focus group phase, sixteen participants were recruited (nine females and seven males aged over eighteen years). The selection criteria were mainly that the participants use at least one social network account and that the selfdeclaration of frequent procrastination on their SNS. In this phase, participants were divided into two sessions to ensure that each session had no more than eight participants. The focus group discussions were based on three main themes with the aim to fill the gap that has been identified in the literature regarding providing more details about the nature of the relationship between procrastination and SNS. These questions were used as a starting point for the discussion. Literature and explanatory scenarios were also provided.

- What is your general perception of procrastination and social media?

- What are the different types of procrastination on SNSs and the users' motivation for facilitating procrastination?

- What role do the design features of SNSs play in persuading procrastination?

\section{B. Diary study for detailed and contextualised diagnosis}

Based on the findings of the focus group, we designed a diary study. This phase sought to confirm the focus group findings and obtain additional information regarding how SNS features may facilitate user procrastination. In this phase, we employed the same participants who had already taken part in the focus group sessions which helped to ensure that data bias was avoided. Consequently, the participants who took part in this phase were already immersed with the research problem. Participants were required to record their stories when they felt they were procrastinating and to share them with us. The diary study benefited from using this method because it involved the participants continually reporting their thoughts, feelings and behaviour in real time and in different contexts [11].

\section{Interviews for clarification}

Three participants from the diary study were called back mainly to clarify what they had reported in their diaries when that was not clear. These interviews helped to enhance the credibility of the analysis through being a validation tool, i.e. to check with them whether we analysed their diary input correctly.

\section{Data analysis}

Thematic analysis was used in order to gain a deeper insight into the data collected via the focus group, diary data and interviews. The thematic analysis approach is widely used for data analysis in qualitative research. It is used as a method to identify, analyse and report patterns. These patterns are important to the description of the phenomena. We followed the steps suggested in [12] to conduct the analysis.

\section{PROCRASTINATION AND SNS DESIGN FINDINGS}

In this section, we categorise procrastination on SNS into four distinct types: avoidance, escapism, emergence and mood modification. Moreover, we reveal procrastination triggers as well as the SNS features that facilitate and persuade procrastination. The findings are summarised in Fig.1.

\section{A. Procrastination on SNS: General Perception}

Participants indicated that they usually use SNS, and eventually procrastinate, to relieve stress and refresh their mood. However, they were receptive to receiving softwareassisted support to gain more control of their procrastination. Participants, who were generally students, acknowledged that procrastination on SNS has a significant effect on their academic performance and it is generally seen as time wasting. Furthermore, participants noted the fine line between procrastination as a way to relieve stress and it being seriously harmful behaviour. One participant stated that "procrastination may be a good thing if it relieves stress because this could improve the quality of work produced but it should be well controlled." In addition, people who are affected by their peer procrastinators claim that procrastinating may become contagious, especially if they are in the same place. One participant said: "If someone procrastinates [on SNS], they may distract others, especially those in the same class" deterring them from doing their primary task and encouraging further procrastination.

\section{B. Procrastination on SNS: Types}

1) Avoidance can be defined as the act of keeping away from or not doing something that needs to be done. In the case of procrastination, avoidance is what people are often inclined to do when they face unpleasable or unenjoyable tasks by delaying starting to work on them [13]. Based on what was derived from the analysis, the lack of motivation to start doing a task might mean avoidance. Lack of motivation can occur because of an individual's limited intrinsic or extrinsic motivation to perform a task. However, the use of SNSs could be more intrinsically motivating and more enjoyable than the original delayed task. Unlike traditional tasks and interactions, SNS can provide instant rewards for users which increase their extrinsic motivation to stay online. For example, when a user posts a photo they might get instant rewards such as likes or positive comments. This reward might persuade users for to continue the usage of SNS and avoid the priority task. A participant said that "the comments and notifications are designed to attract people to stay online" without considering 


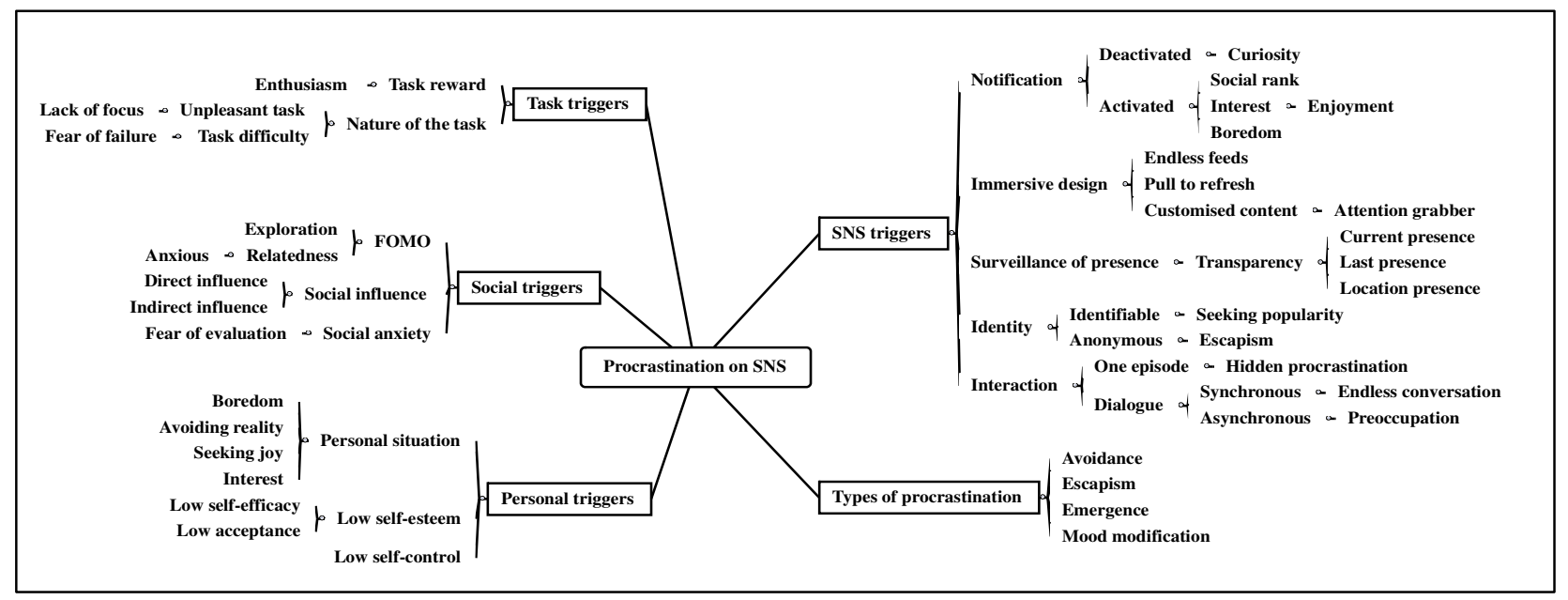

Fig.1: Procrastination types and triggers

whether this will then encourage them to ignore their original tasks and jobs.

2) Escapism can happen when ignoring real-life problems, anxiety and depression by immersing in SNS [14]. In some cases, such escapism is correlated with procrastination. Some people tend to delay or avoid dealing with financial difficulties, loneliness or fear through being an alternative, often unreal, environment online [15]. One of the triggers is the feeling of low acceptance from peers during a group activity which encourages users to share ideas on SNSs and get better response. SNS features allow and probably persuade users to customise their profile and create a list of people who they like to follow, i.e. the echo chambers through collaborative filtering algorithms applied to news and posts. This technique helps to build an alternative reality consisting only from what a person like to hear and see and this makes SNS and ideal escape environment for users who experience negative feelings in their real lives. Some participants argued that SNSs allow them to have anonymous accounts and that anonymity encourages them to express their real self without being identifiable by others. This persuades users to stay active for a long time even if being active has a negative impact on other important tasks. Some users preferred their alternative online life to their real life, and those people tend not to perceive time spent on SNSs as procrastination. Instead, they may feel procrastination if they do not fulfil the task of their online persona, e.g. gamers gathering online for a virtual battle.

3) Emergence type of procrastination can happen when people focusing on a task such as attending a lecture or writing a paper, get distracted by SNS task, which then triggers procrastination online. The emerged task might lead people to stop focusing or delay work on the original task, which harms the productivity of their work [16]. With SNS being a continuous medium for communication, there is always a threat to get distracted. One of the participants reported that: "the notification is the worst feature that leads me to check and then procrastinate when I am focussing on my work'.

4) Mood modification type of procrastination occurs when a user procrastinates to experience a better feeling. Mood modification is also, one of the six core components of behavioural addiction, including addiction to SNS, which has been considered by [17]. Mood modification refers to the subject experience that people report as a result of engaging in a particular activity and can be seen as a coping strategy [17]. However, mood modification in SNS occurs when a person visits SNSs to change their mood instead of working on their tasks. For example, when a user thinks that there might be some stress associated with a task they need to perform, they might start accessing SNSs in order to relieve the stress and build some confidence. One participant said: "while I was working I felt stressed so I decided to have a look on Facebook" and comparing the high level of joy on SNS with the stress that is associated with the task, users find it challenging to stop procrastinating. One participant commented: "even when I realised I was procrastinating and, it was hard to stop".

\section{Personal triggers}

This kind of persuasion trigger refers to the personal context and can be divided into three sub-categories; personal situation, low self-esteem, and low self-control.

1) Personal situation refers to personal needs, feelings and emotions that lead users to procrastinate. Personal situations are divided into four sub-categories which include boredom, avoiding reality, seeking joy, and interest. Boredom is widely believed to be an important trigger of procrastination because most of the participants reported that they tend to procrastinate to combat a boring, not necessarily challenging, task and change their mood. A participant said: "There was no reason for procrastinating other than that I was feeling bored so chose to go on Instagram'. Avoiding reality happens when a person faces a situation that they do not believe they are able 
to deal with it due to low self-efficacy. They then start accessing SNSs and avoid working on it. For example, a participant said "I was browsing YouTube to see some funny videos instead of working on an important task that need full concentration that I find difficult',

The emotional need for joy, i.e. seeking joy plays an important role in explaining people's procrastination. SNSs provide their users with different types of activities that might deliver high levels of enjoyment such as chatting with friends, keeping up to date with their fans and sharing good moments with others. In addition, interest is associated with a personal situation and can trigger procrastination. SNSs can be persuasive through customising and personalising the content based on user interest in order to seize their attention for more SNSs usage which can result in procrastination. For example, hashtags are presented to the user to match their recent search and browsing.

2) Low Self-esteem can be defined as the negative evaluation of oneself, or abilities [18]. Low self-esteem has been found to be one of the procrastination triggers that often leads people to avoid or delay performing a task [19]. Low self-esteem is divided to two subcategories which include low self-efficacy and low acceptance. Self-efficacy is defined as an individual belief that someone will be able to accomplish a specific task. An essential component to achieving something is our confidence that we can achieve it [20]. There is evidence that low self-efficacy has been found as one of the predictors of procrastination [21, 22]. For example, when SNS user has low confidence to perform a task like sharing ideas during class time, this may encourage them to avoid focusing on the class and tend to procrastinate via SNSs. Some participants noted that 'People use SNSs to express their ideas under a nickname when they are not confident enough about what they are sharing".

Low acceptance refers to the feeling of not being recognised or appreciated by others. Participants claimed that SNS allow them to share whatever they want without feeling any pressure, they get a level of satisfaction from their friends on SNSs, and they feel less inhibited or incompetent. Moreover, for people who have low relatedness and feelings of not being accepted by others, SNS may meet their needs and allow them to create relationships and find new virtual friends. Hence, they might feel appreciated, understood and less lonely without looking at the meaning of that in practical terms, i.e. their job and reality. For example, some participants mentioned that while being a teenager, she used to "use SNSS to be accepted by other peers',

3) Low self-control has an association relationship with procrastination $[23,24]$. A study that explored the relationship between low self-control and Facebook claimed that the enjoyment of browsing Facebook makes students lose track of time and that can causes delays to tasks that needed to be done such as writing a paper or preparing for an exam [25]. However, most of the diary and focus group participants noted that users who have low self-control typically find it difficult to stop accessing SNSs due to the level of enjoyment and temptation that SNSs provide. This enjoyment can be achieved as a result of persuasive features like customised and personalised content which trigger interest or stimulate curiosity for new information.

\section{Social triggers}

Social triggers denote the family of triggers related to the users' human relationships and interests. Social triggers are divided into three sub-categories: fear of missing out (FOMO), social influence, and social anxiety.

1) FOMO has been defined as a desire to stay continually connected with what others are doing to avoid missing important events and content [26]. However, the strong desire to keep up to date with what is happening on SNSs and what people do seems to be one of the most important triggers of procrastination. Hence, users start to access SNSs to ensure that they do not miss anything. Participants mentioned some SNS features are much persuasive and lead them to procrastinate such as Snapchat stories and users' status, which are temporarily valid, i.e. scarcity [27]. One participant said: "Snapchat stories encourage me to read them because they will disappear after 24 hours', Exploration features can also be associated with FOMO, and these features tempt users to explore and seek more information about particular content. For example, when a user checks their SNSs accounts to avoid missing any information, they usually start to explore more information related to what they started to explore. A participant said "I was watching video on YouTube and then other videos appeared, so I watched a few more".

2) Social influence might persuade users to procrastinate either directly or indirectly. Indirect influence happens when people normalise checking SNS because others are doing the same. One participant said: "seeing others browsing Facebook during the class encouraged me to do so". Direct influence can occur when somebody asks other peers, in person or online, to access SNS for interesting content, e.g. via tagging them in a picture or a post.

3) Social anxiety can lead SNS users to procrastinate mainly because of the fear of evaluation. Fear of evaluation is maximised when users have low self-efficacy and limited confidence which adversely affect their intrinsic motivation to perform the task. SNS enable users to personalise their accounts by organizing a list of followers and banding together those who have inconsistent views which helps to avoid having negative reactions [28].

\section{E. SNSs features triggers}

1) Notification features can be described as enhancing connectivity and up-to-datedness via user alerts when a user interacts with particular services [29]. Most of our participants agreed that notifications distracted them while they are working on a particular task and encouraged them to start accessing SNS. Therefore, notification seems to be the first trigger that usually facilitates procrastination. Notification can 
lead to procrastination in its two modalities; deactivated and activated notification. Deactivated notifications seem to persuade users to procrastinate indirectly because they are more tempted to know whether they received messages when a notification is muted. This may trigger user curiosity to access SNS. Hence, when the user browses SNSs they tend to compensate and perform other activities, such as chatting or searching for information. In contrast, an activated notification may trigger user procrastination directly. Thus, clicking on the notification will lead users to start accessing SNSs and other activities might emerge. One participant said: "I started browsing more and procrastinating when I got a notification through promoting a discount code for online clothes". We argue that there are other creative ways a notification may solve the aforementioned dilemma, e.g. collecting notification from different social networks in one place to demotivate further browsing and providing a recap-like notification which will be a way in the middle between active and deactivated notification.

2) Immersive design; can lead users to stay online for a long time because of the fear of missing temporal content such as snap chat stories. Moreover, an SNS feature such as endless feeds could persuade users to procrastinate while using SNSs by simply continuing to view other posts. For example, Twitter's never-ending newsfeed offers continuous browsing for extended periods of time. One participant said: "I continued to procrastinate just scrolling through Twitter's home page". The, Pull to refresh feature is designed to constantly remind users of the perpetual nature of newsfeeds. For example, when a user updates their newsfeed on Twitter, the "pull to refresh" icon appears, increasing the expectation of seeing something new, even if nothing comes up. This feature persuades users to stay online even for a matter of seconds to see what could follow. In addition the customized content feature aims to provide content based on the user's experience or their previous browsing history profile. This also occurs by providing links or alternative content linked to the content that a user is viewing at a given time. Hence, alternative content may trigger users' interest to continue viewing content, increasing the overall user usage. One participant reported that: "When I watch a video I find others on the recommended list that attracted me".

3) Identity features are the features that allow users to profile and represent themselves, e.g. choosing a name, avatar, status, motto and profile picture [7, 29]. Some users tend to use real information to represent themselves, whereas others prefer to use made-up identities. Users who use identifiable or real information might feel pressured to spend more time and "gain more popularity and social influence". Craving popularity, in turn, triggers continuous usage in order to gain more followers which leads to further procrastination. In other cases, user popularity facilitates financial gain from advertising products or even by proliferating the popularity of other users. Therefore, a user might stay active for longer in order to satisfy their followers and increase their account reputation. SNS features allow users to increase their popularity by showing popularity information on other followers, i.e. social proof [27], thus increasing the popularity competition between them. Anonymous users prefer to use fictitious information to represent themselves on SNSs for reasons, such as privacy and freedom to express views which might be controversial, or being more relaxed and comfortable about their online presence [30]. One participant noted that: "Using a nickname increases the users' confidence to talk without being worried because they are anonymous" and this leads to an extensive time on SNS as well.

4) Presence features refer to the extent to which users know if others are available [7]. Using presence features, people can share some details about their location or the times they are active. Surveillance of presence feature causes procrastination by broadcasting the user's status as online and active. Therefore, once the user starts accessing SNSs, they may feel they are being monitored by others, increasing the pressure to respond to any request from their contacts or followers. A participant said "because two blue ticks appeared I'm forced to reply' which means she felt that the system was monitoring her presence which forced her to spend some time chatting at the expense of her work. Similarly, SNSs monitor their users through the feature of transparency, which captures information about their SNS access history which includes current presence, last presence and user location.

Current presences feature identifies if a user's contacts are currently online. Some participants claimed that appearing online when they receive a message forces them to reply to these messages in order to maintain good relations with their friends and avoid losing them. One participant said: "because I appeared online I had to start the conversation'. Moreover, while the user appears online this may trigger starting a conversation because other users may expect that the user is available at that moment. This expectation adds pressure on users to reply which can result in procrastination. Similarly, last presence feature adds pressure on users by encouraging them to access SNSs to respond to and interact with others. For example, when a user's status shows that they were recently active, it provides an indication to others and increases their expectations of receiving a response. This expectation could have a significant effect on user procrastination by enticing responses to message request in an attempt to maintain good relations with other users. A participant said: "I procrastinated on WhatsApp as I opened my friend's message page and closed it without replying to her messages which means that two blue ticks appeared to her'. Furthermore, the presence of user location might also facilitate procrastination. For example, when a user posts a photo on Instagram, their location appears to others. Hence, the location information might trigger further interest in posts originating from the same location, triggering further usage.

5) Interaction features on SNSs are the features that allow users to interact with others. Several research studies suggest 
that the main motivation for users to be active on SNSs is to use the features that allow them to interact with their peers $[31,32]$. These features were divided into two subcategories based on the nature of the interaction as one episode and dialogue. One episode interaction refers to a single, nonrepeatable interaction, such as liking a post or re-tweeting. This type of feature is seen as a procrastination trigger because users keep checking their SNSs in order to see how many likes or re-tweets they have received, especially those interested in their level of popularity. One of the participants said: "I posted a photo about my trip to Paris and I kept thinking about the number of likes I got for it, so I couldn't focus on my work". Hence, the one episode feature enables users to get a social reward which increases their extrinsic motivation to frequently check for more reward. Seeking popularity may associate users to procrastinate as SNSs allow users to count how many likes and retweets they have gained on each post. Moreover, one episode features may trigger user curiosity about their own post. For example, when a user posts a photo on Instagram, they may start thinking about user feedback, both positive and negative feedback. Over time, the user may begin to feel worried why others are ignoring the post if no one engages with it, which in turn can lead to the emergence of hidden procrastination. On the other hand, when the user receives feedback on their post, such as likes or comments, this may lead to further posts in order to increase their followers' satisfaction and gain more popularity.

Dialogue feature refers to continuing interaction between SNS users, such as chatting features, and comment sections. This type of interaction may increase procrastination as many participants of the focus group and the diary study reported that chatting with others increased their SNS usage which leads them to procrastinate. Interaction within a dialogue can be divided into two sub categories: synchronous and asynchronous. In the synchronous dialogue, the nature of the interaction requires both users to interact with each other at the same time, which makes users more motivated to keep interacted due to the frequency of responses. A participant said: "I continued to chat with him for more than half hour". Therefore, users who have low self-control tend to procrastinate more because they find it difficult to stop the interaction which can result in seemingly endless conversations. The threat of endless conversations is increased when the user expects a quick reply and the feature shows that the other person is "typing" or "recording." This may increase user expectations to see something new which triggers continued usage. In contrast, asynchronous refers to communication in which data can be transmitted intermittently rather than in a steady stream which can lead users to be preoccupied by thinking about received responses.

\section{F. Task triggers}

Task triggers have been identified as one of the procrastination predictors [33]. Some people might tend to procrastinate because of the nature of the task. For example, the task itself is unenjoyable, stressful or frustrating which prevents them from dealing with it or might lead to lack of focus. Also, task difficulty can lead SNS users to avoid performing a given task because of the fear of failure. At the same time, the reward of a task may have a significant effect on how individuals perform a task, and the reward can be intrinsic or extrinsic. Intrinsic motivation can be defined as engaging in a task for the reward inherent in the task such as interest and enjoyment, whereas extrinsic motivation refers to performing an action to obtain a reward or outcome[34]. The task reward has a significant effect on user's enthusiasm to perform the task.

\section{CONCLUSION}

In this paper, we elaborated on our initial observation about the role of social media design in triggering procrastination. Our study showed that the design of SNS features can, in some context of usage, play an important role in persuading users to procrastinate, directly and indirectly. We argue that it is within the social responsibility of SNS companies to aid people to stay in control of their usage and offer tools to help them combat procrastination. Steps in this direction started to be taken, e.g. iOS Screen Time and Google Digital Wellbeing programs. However, these tools are mainly focused on the interaction between the user and the device, e.g. managing time spent, content reviewer and setting notification preferences. However, our study showed that the reasons for procrastination are inherent to the way social interaction is facilitated through social media, e.g. peer pressure and social influence, and personality of the user, e.g. low self-control. This calls for more profound persuasive tools and personalisation of procrastination countermeasure to fit the user's personal and social context.

\section{REFERENCES}

[1] K. B. Klingsieck, "Procrastination: When good things don't come to those who wait," European Psychologist, vol. 18, p. 24, 2013.

[2] W. J. Knaus, "Overcoming procrastination," Rational Living, 1973.

[3] M. Beheshtifar, H. Hoseinifar, and M. Moghadam, "Effect procrastination on work-related stress," European Journal of Economics, Finance and Administrative Sciences, vol. 38, pp. 59-64, 2011.

[4] L. Ivarsson and P. Larsson, "Personal Internet usage at work: A source of recovery," Journal of Workplace Rights, vol. 16, 2011.

[5] K. R. Kim and E. H. Seo, "The relationship between procrastination and academic performance: A meta-analysis," Personality and Individual Differences, vol. 82, pp. 26-33, 2015.

[6] D. J Kuss, M. D Griffiths, L. Karila, and J. Billieux, "Internet addiction: a systematic review of epidemiological research for the last decade," Current pharmaceutical design, vol. 20, pp. 4026-4052, 2014.

[7] J. H. Kietzmann, K. Hermkens, I. P. McCarthy, and B. S. Silvestre, "Social media? Get serious! Understanding the functional building blocks of social media," Business horizons, vol. 54, pp. 241-251, 2011.

[8] D. Blackwell, C. Leaman, R. Tramposch, C. Osborne, and M. Liss, "Extraversion, neuroticism, attachment style and fear of missing out as predictors of social media use and addiction," Personality and Individual Differences, vol. 116, pp. 69-72, 2017.

[9] B. J. Fogg, "Persuasive technology: using computers to change what we think and do," Ubiquity, vol. 2002, p. 5, 2002.

[10] A. Alblwi, A. Stefanidis, K. T. Phalp, and R. Ali, "Procrastination on Social Networking Sites: Combating by Design," IEEE 13th International Conference on Research Challenges in Information Science RCIS, 2019. 
[11] R. C. Fraley and N. W. Hudson, "Review of intensive longitudinal methods: an introduction to diary and experience sampling research," ed: Taylor \& Francis, 2014.

[12] V. Braun and V. Clarke, "Using thematic analysis in psychology," Qualitative research in psychology, vol. 3, pp. 77-101, 2006.

[13] J. R. Ferrari, J. O'Callaghan, and I. Newbegin, "Prevalence of Procrastination in the United States, United Kingdom, and Australia: Arousal and Avoidance Delays among Adults," North American Journal of Psychology, vol. 7, 2005.

[14] M. Griffiths, "Does Internet and computer" addiction" exist? Some case study evidence," CyberPsychology and Behavior, vol. 3, pp. 211-218, 2000.

[15] H. Warmelink, C. Harteveld, and I. Mayer, "Press Enter or Escape to Play-Deconstructing Escapism in Multiplayer Gaming," in DiGRA Conference, 2009.

[16] G. Mark, S. Iqbal, M. Czerwinski, and P. Johns, "Focused, aroused, but so distractible: Temporal perspectives on multitasking and communications," in Proceedings of the 18th ACM Conference on Computer Supported Cooperative Work \& Social Computing, 2015, pp. 903-916.

[17] M. D. Griffiths, D. J. Kuss, and Z. Demetrovics, "Social networking addiction: An overview of preliminary findings," in Behavioral addictions, ed: Elsevier, 2014, pp. 119-141.

[18] K. D. Neff, "Self - compassion, self - esteem, and well - being," Social and personality psychology compass, vol. 5, pp. 1-12, 2011.

[19] S. J. Scher and J. R. Ferrrari, "Toward an understanding of academic and nonacademic tasks procrastinated by students: The use of daily logs," Psychology in the Schools, p. 359, 2000.

[20] A. Bandura, "Self-efficacy: toward a unifying theory of behavioral change," Psychological review, vol. 84, p. 191, 1977.

[21] P. Steel, "The nature of procrastination: A meta-analytic and theoretical review of quintessential self-regulatory failure," Psychological bulletin, vol. 133, p. 65, 2007.

[22] C. A. Wolters, "Understanding procrastination from a self-regulated learning perspective," Journal of Educational Psychology, vol. 95, p. 179, 2003.
[23] R. J. Lee-Won, L. Herzog, and S. G. Park, "Hooked on Facebook: the role of social anxiety and need for social assurance in problematic use of Facebook," Cyberpsychology, Behavior, and Social Networking, vol. 18, pp. 567-574, 2015.

[24] K. Wilson, S. Fornasier, and K. M. White, "Psychological predictors of young adults' use of social networking sites," Cyberpsychology, Behavior, and Social Networking, vol. 13, pp. 173-177, 2010.

[25] L. D. Rosen, L. M. Carrier, and N. A. Cheever, "Facebook and texting made me do it: Media-induced task-switching while studying," Computers in Human Behavior, vol. 29, pp. 948-958, 2013.

[26] A. K. Przybylski, K. Murayama, C. R. DeHaan, and V. Gladwell, "Motivational, emotional, and behavioral correlates of fear of missing out," Computers in Human Behavior, vol. 29, pp. 1841-1848, 2013.

[27] R. B. Cialdini, Influence vol. 3: A. Michel Port Harcourt, 1987.

[28] H. Oinas-Kukkonen and M. Harjumaa, "Persuasive systems design: Key issues, process model, and system features," Communications of the Association for Information Systems, vol. 24, p. 28, 2009.

[29] N. I. M. Dawot and R. Ibrahim, "A review of features and functional building blocks of social media," in Software Engineering Conference (MySEC), 2014 8th Malaysian, 2014, pp. 177-182.

[30] R. Kang, S. Brown, and S. Kiesler, "Why do people seek anonymity on the internet?: informing policy and design," in Proceedings of the SIGCHI Conference on Human Factors in Computing Systems, 2013, pp. 2657-2666.

[31] N. M. Suki, T. Ramayah, and K. K. Ly, "Empirical investigation on factors influencing the behavioral intention to use Facebook," Universal Access in the Information Society, vol. 11, pp. 223-231, 2012.

[32] A. D. Smock, N. B. Ellison, C. Lampe, and D. Y. Wohn, "Facebook as a toolkit: A uses and gratification approach to unbundling feature use," Computers in Human Behavior, vol. 27, pp. 2322-2329, 2011.

[33] T. A. Pychyl, J. M. Lee, R. Thibodeau, and A. Blunt, "Five days of emotion: An experience sampling study of undergraduate student procrastination," Journal of social Behavior and personality, vol. 15, p. 239, 2000.

[34] R. M. Ryan and E. L. Deci, "Self-determination theory and the facilitation of intrinsic motivation, social development, and well-being," American psychologist, vol. 55, p. 68, 2000. 